\title{
Objective Olfactory Findings in Hospitalized Severe COVID-19 Patients.
}

Jerome Lechien ${ }^{1}$, Morgane Ducarme ${ }^{2}$, Sammy Place ${ }^{2}$, Carlos Chiesa-Estomba ${ }^{3}$, Mohamad Khalife $^{2}$, Giacomo De Riu ${ }^{4}$, Luigi Vaira ${ }^{4}$, Christophe de Terwangne ${ }^{5}$, Shahram Machayekhi $^{2}$, Arnaud Marchant ${ }^{6}$, Fabrice Journe ${ }^{1}$, and Sven Saussez ${ }^{1}$

${ }^{1} \mathrm{UMONS}$

${ }^{2}$ Hospital Centre EpiCURA

${ }^{3}$ Universidad San Sebastian

${ }^{4}$ Sassari University Hospital

${ }^{5}$ Centre Hospitalier EpiCURA asbl

${ }^{6} \mathrm{ULB}$

May 26, 2020

\section{Objective Olfactory Findings in Hospitalized Severe COVID-19 Patients.}

Jerome R. Lechien, MD, PhD, MS ${ }^{1-4}$, Morgane Ducarme, MD ${ }^{5}$, Sammy Place, MD ${ }^{6}$, Carlos M. ChiesaEstomba, MD, $\mathrm{MS}^{1,7}$, Mohamad Khalife, $\mathrm{MD}^{1,8}$, Giacomo De Riu, MD ${ }^{9}$, Luigi Angelo Vaira, $\mathrm{MD}^{9}$, Christophe de Terwangne, $\mathrm{MD}^{10}$, Shahram Machayekhi, $\mathrm{MD}^{10}$, Arnaud Marchant, MD, PhD ${ }^{11}$, Fabrice Journe, $\mathrm{PhD}^{2^{*}}$, Sven Saussez, MD, $\mathrm{PhD}^{1,2,4^{*}}$

\section{Institutions:}

1. COVID-19 Task Force of the Young-Otolaryngologists of the International Federations of Oto-rhinolaryngological Societies (YO-IFOS).

2. Department of Human Anatomy and Experimental Oncology, Faculty of Medicine, UMONS Research Institute for Health Sciences and Technology, University of Mons (UMons), Mons, Belgium

3. Department of Otolaryngology-Head \& Neck Surgery, Foch Hospital, School of Medicine, UFR Simone Veil, Université Versailles Saint-Quentin-en-Yvelines (Paris Saclay University), Paris, France.

4. Department of Otorhinolaryngology and Head and Neck Surgery, CHU de Bruxelles, CHU Saint-Pierre, School of Medicine, Université Libre de Bruxelles, Brussels, Belgium.

5. Department of Surgery, EpiCURA Hospital, Hornu, Belgium.

6. Department of Internal Medicine, EpiCURA Hospital, Baudour, Belgium.

7. Department of Otorhinolaryngology-Head \& Neck Surgery, Hospital Universitario Donostia, San Sebastian, Spain.

8. Department of Otolaryngology-Head \& Neck Surgery, EpiCURA Hospital, Baudour, Belgium.

9. Maxillofacial Surgery Unit, University Hospital of Sassari, Sassari, Italy.

10. Department of Intensive Care, EpiCURA Hospital, Hornu, Belgium.

11. Institute for Medical Immunology, Université libre de Bruxelles, Brussels, Belgium.

* Dr Saussez \& Dr Journe have equally contributed to this work and should be regarded as joint senior authors.

Running title: Coronavirus and Olfaction. 
Acknowledgments: The workers of the Anatomy Lab of UMONS who helped to collect data.

Conflict of interest statement : The authors have no conflicts of interest

\section{Correspondence to:}

Dr. Jay R. Lechien, M.D., Ph.D., M.S.

Department of Otorhinolaryngology and Head and Neck Surgery, Foch Hospital, School of Medicine, UFR Simone Veil, Université Versailles Saint-Quentin-en-Yvelines (Paris Saclay University), Paris, France.

Email: Jerome.Lechien@umons.ac.be

Telephone: +3265373584

\section{To The Editor}

Since the onset of the coronavirus disease 2019 (COVID-19) pandemic, many patients reported sudden loss of smell (SLS).1 However, due to the sanitary situation, only a few studies investigated SLS with objective testings, which remains essential to confirm the olfactory dysfuntion.2-4 All these studies involved outpatients with mild COVID-19 forms. The mean age and the prevalence of comorbidities were low,2-4 leading some authors to suspect that SLS could be more specific to mild COVID-19 forms. 5 In this study, we investigated the prevalence of self-reported and objective SLS in severe COVID-19 patients.

\section{Methods:}

Adults (33-88 years old) with severe COVID-19 were recruited from the Department of Medicine of the EpiCURA Hospital (Hornu, Belgium; Ethics Committee: EpiCURA-2020-2303). The disease was confirmed through nasopharyngeal swab (RT-PCR). Patients were defined as severe COVID-19 if they required continuous care (oxygenotherapy, blood pressure monitoring) in internal medicine or intensive care units.

Patients with neurological disorder, chronic rhinosinusitis or history of nasal surgery prior the pandemic were excluded. Epidemiological and clinical data were collected at the hospital discharge. Details of the patient-reported outcome questionnaire used for data collection are reported in a previous study. 3 Briefly: 1) olfactory and gustatory questions were based on the smell and taste component of the National Health and Nutrition Examination Survey; 2) symptoms were evaluated through a 4-point scale ranging from 0 (no symptom) to 4 (severe symptoms);3 3) nasosinusal symptoms were evaluated through the French sinonasal outcome test-22 (SNOT-22).6Patients benefited from psychophysical olfactory evaluation through sniffin'stick tests (Medisense, Groningen, Netherlands): 16 pens were presented to patients every 30 seconds. The patient had to choose the adequate term describing the smell between 4 given options. The test was scored on a total of 16 points and allowed categorization into in 3 groups: normosmia (score between 12-16), hyposmia (score between 9-11) and anosmia (score <9).3 Moreover, the following hospitalization outcomes were recorded: duration of hospitalization (days); admission biology (D-dimer; hemoglobin; leucocyte count; lymphocyte count; CRP; creatitin; bilirubin; platelet count; LDH; $\left.\mathrm{Na}^{+} ; \mathrm{K}^{+} ; \mathrm{Cl}^{-}\right)$; 1-month serology $(\operatorname{IgG})$ and chest computed tomography findings. Subjective and objective evaluations were made meanwhile.

The relationship between clinical and olfactory outcomes was analyzed through multiple linear regression between scale variables and through Mann-Whitney test and boxplot representation for groups versus scale variables (SPSS, v22,0; IBM-Corp, Armonk, NY, USA).

\section{Results:}

Complete evaluation was performed in 47 patients, including 25 females. Patients were hospitalized in EpiCURA hospital from March, 20th, 2020 to April, 16th, 2020. Evaluations were conducted 41.0 \pm 10.3 days after the onset of symptoms, corresponding to 1-2 weeks after the end of the hospitalization. Clinical outcomes are reported in Table 1. The most prevalent symptoms were: fever, asthenia and anorexia. The mean duration of symptoms before hospitalization was $10.7 \pm 5.0$ days. Eight patients were hospitalized in 
intensive care unit (ICU) for a mean duration of $8.5 \pm 5.6$ days. The CT-scan and blood tests features are reported in Table 1.

Psychophysical olfactory evaluations indicated that 4 (8.5\%) and $9(19.1 \%)$ patients reported anosmia and hyposmia (in the entire cohort), respectively (Table 2 ). Note that three hyposmic patients reported in the patient-reported outcome questionnaire that they have hyposmia prior the infection. Excluding these three patients, the prevalence of objective SLS in our cohort was $21.3 \%$.

Eight and 10 patients experienced -subjective- total and partial loss of smell, respectively, over the clinical course of the disease; accounting for $38.3 \%$ of individuals. Among them, only 3 and 4 were anosmic and hyposmic (38.9\%), respectively. The three patients who experienced hyposmia prior the pandemic were not included in the subjective SLS patients. According to subjective evaluations of olfaction, $38.3 \%$ of patients complained from SLS. Additional olfactory outcomes are reported in Table 2.

Patients with diabetes had lower sniffin'sticks test results compared with those without diabetes (Mann Whitney $\mathrm{U}$ test; $\mathrm{p}=0.045)$. The linear regression analyses revealed significant negative associations between the sniffin'sticks test and age $\left(\mathrm{r}_{\mathrm{s}}=-0.339 ; \mathrm{p}=0.032\right)$. Symptom duration was significantly correlated with the severity of fever $\left(\mathrm{r}_{\mathrm{s}}=0.395 ; \mathrm{p}=0.046\right)$ and dysphonia $\left(\mathrm{r}_{\mathrm{s}}=0.572 ; \mathrm{p}=0.002\right)$. Duration of hospitalization was significantly correlated with age $\left(r_{s}=0.402 ; p=0.008\right)$. Serum IgG concentration measured by the SARS-CoV2 LIAISON? test (Diasorin, Centralino, Italy) was negatively correlated with the severity of nasal burning $\left(\mathrm{r}_{\mathrm{s}}=-0.407 ; \mathrm{p}=0.029\right)$.

\section{Discussion:}

Olfactory disorder is undoubtedly a key symptom of mild COVID-19 patients, affecting more than $70 \%$ of patients.4,5 However, its prevalence remains uninvestigated in severe forms of the disease. In this study, we found that $38.3 \%$ of patients with severe disease experience SLS. Among them, $38.9 \%$ had abnormal objective tests 1-month after the onset of the infection. Irrespective to the method used to evaluate the prevalence of SLS (patient-reported outcome questionnaireversus objective tests), these data indicate that SLS could be more prevalent in mild-to-moderate forms of the infection.

According to a previous study conducted in the same population and with the same methods, self-reported SLS concerned more than $70 \%$ of mild COVID-19 patients and, among them, $62 \%$ had abnormal objective evaluations. 3 The higher incidence of SLS in mild forms of COVID-19 suggests a relative compartmentalization of the disease. Such compartmentalization may involve differences in immune responses to SARS-CoV-2 at the level of nasal and olfactory mucosa. In patients with potent mucosal immune responses, viral replication and dissemination to the lower respiratory tract may be better controlled and this could be at the expense of local inflammation and symptoms involving nasal and bulb regions. In patients with less potent mucosal immune responses, viral replication could spread to the lower respiratory tract and lead to systemic immune response and inflammation. This hypothesis is supported by our observation that nasal burning was inversely correlated with SARS-CoV-2 serum IgG whereas severe forms of the disease have been positively correlated with SARS-CoV-2 IgG responses.7 Further studies are needed to test this hypothesis. Both age and diabetes could be favoring factors in the development of SLS, which is well known in other olfactory diseases. 8,9

The main limitations of the present study are the low number of patients and the performance of olfactory tests one month after the onset of symptoms. Performing the tests during hospitalization was difficult due to the sanitary situation, the patient clinical state, and the difficulties to correctly sense the pens with transnasal oxygenation. Although this possibility is not supported by patient-reported symptoms, the delay between the onset of symptoms and the objective olfactory testing may underestimate the incidence of olfactory dysfunction.

\section{Author contribution:}

Study concept and design: Lechien, Ducarme, Chiesa, Saussez, De Rui, Vaira. 
Acquisition, analysis, or interpretation of data: Lechien, Saussez, Khalife, Place, Ducarme, Machayekhi, de Terwangne.

Drafting of the manuscript: Lechien, Saussez, Journe, Marchant.

Critical revision of the manuscript for important intellectual content: Saussez, Chiesa, Journe, De Riu, Vaira, Marchant.

Funding : FRMH Funding.

Competing interests : None. Sponsorships: None. Funding source: None.

\section{References:}

1. Lechien JR, Chiesa-Estomba CM, De Siati DR, et al. Olfactory and gustatory dysfunctions as a clinical presentation of mild-to-moderate forms of the coronavirus disease (COVID-19): a multicenter European study. Eur Arch Otorhinolaryngol . 2020. doi: 10.1007/s00405-020-05965-1.

2. Moein ST, Hashemian SMR, Mansourafshar B, Khorram-Tousi A, Tabarsi P, Doty RL. Smell dysfunction: a biomarker for COVID-19. Int Forum Allergy Rhinol . 2020. doi: 10.1002/alr.22587. 3. Lechien JR, Cabaraux P, Chiesa-Estomba CM, et al. Objective olfactory evaluation of self-reported loss of smell in a case series of 86 COVID-19 patients. Head Neck . 2020 May 21. doi: 10.1002/hed.26279.

4. Angelo Vaira L, Hopkins C, Salzano G, et al. Olfactory and gustatory function impairment in COVID19 patients: Italian objective multicenter-study. Head Neck . 2020. doi: 10.1002/hed.26269.

5. Lechien JR, Chiesa-Estomba CM, Hans S, Barillari MR, Jouffe L, Saussez S. Loss of Smell and Taste in 2,013 European Mild-to-Moderation COVID-19 Patients. Accepted for publication in Ann Int Med . 2020.

6. de Dorlodot C, Horoi M, Lefebvre P, Collet S, Bertrand B, Eloy P, Poirrier AL. French adaptation and validation of the sino-nasal outcome test-22: a prospective cohort study on quality of life among 422 subjects. Clin Otolaryngol . 2015; 40(1):29-35. doi: 10.1111/coa.12315.

7. Vabret N, Britton GJ, Gruber C et al. Immunology of COVID-19: current state of the science. Immunity . 2020. https://doi.org/10.1016/j.immuni.2020.05.002

8. Zaghloul H, Pallayova M, Al-Nuaimi O, Hovis KR, Taheri S. Association between diabetes mellitus and olfactory dysfunction: current perspectives and future directions. Diabet Med . 2018; 35(1):41-52. doi: $10.1111 /$ dme. 13542 .

9. Doty RL. Age-Related Deficits in Taste and Smell. Otolaryngol Clin North Am . 2018; 51(4):815-825. doi: 10.1016/j.otc.2018.03.014.

Table 1: Epidemiological \& Clinical Characteristics of Patients .

\begin{tabular}{ll}
\hline Characteristics & Patients (N-\%) \\
\hline Age (Mean - SD) - yo & $58.8 \pm 12.9$ \\
Gender (Female/Male) & $25 / 22$ \\
Ethnicity $(N$ - \%) & \\
Caucasian & $44(93.6)$ \\
North African & $2(4.3)$ \\
Black African & $1(2.1)$ \\
Smoker & $0(0)$ \\
Patients with seasonal allergy & $12(25.5)$ \\
Comorbidities & \\
Hypertension & $10(21.3)$ \\
GERD & $9(19.1)$ \\
Hypothyroidism & $9(19.1)$
\end{tabular}




\begin{tabular}{|c|c|}
\hline Characteristics & Patients (N-\%) \\
\hline Diabetes & $7(14.9)$ \\
\hline Asthma & $5(10.6)$ \\
\hline Heart problems & $4(8.5)$ \\
\hline Neurological diseases & $3(6.4)$ \\
\hline Renal failure & $2(4.2)$ \\
\hline Hepatic insufficiency & $1(2.1)$ \\
\hline Untreated cancer & $1(2.1)$ \\
\hline Depression & $1(2.1)$ \\
\hline Autoimmune disease & $0(0)$ \\
\hline Respiratory insufficiency & $0(0)$ \\
\hline \multicolumn{2}{|l|}{ General Symptoms (N-\%) } \\
\hline Asthenia & $44(93.6)$ \\
\hline Fever $(>38 \mathrm{C})$ & $44(93.6)$ \\
\hline Anorexia & $44(93.6)$ \\
\hline Dyspnea & $41(87.2)$ \\
\hline Cough & $38(80.9)$ \\
\hline Myalgia & $36(76.6)$ \\
\hline Headache & $35(74.5)$ \\
\hline Diarrhea & $32(68.1)$ \\
\hline Arthralgia & $27(57.4)$ \\
\hline Chest pain & $26(55.3)$ \\
\hline Nausea/vomiting & $24(51.1)$ \\
\hline Abdominal pain & $22(46.8)$ \\
\hline Conjonctivitis & $14(29.8)$ \\
\hline \multicolumn{2}{|l|}{ Ear, nose and throat Sympotms (N - \%) } \\
\hline Rhinorrhea & $33(70.2)$ \\
\hline Nasal obstruction & $30(63.8)$ \\
\hline Dysphonia & $27(57.4)$ \\
\hline Throat sputum & $26(55.3)$ \\
\hline Postnasal drip & $25(53.2)$ \\
\hline Sore throat & $23(48.9)$ \\
\hline Dysphagia & $21(44.7)$ \\
\hline Face pain/heaviness & $18(38.3)$ \\
\hline Nose burning & $15(31.9)$ \\
\hline Ear pain & $14(29.8)$ \\
\hline Presumed hyposmia & $10(21.3)$ \\
\hline Presumed anosmia & $8(17.2)$ \\
\hline Cacosmia & $8(17.2)$ \\
\hline Taste dysfunction & $6(12.8)$ \\
\hline Phantosmia & $1(2.1)$ \\
\hline \multicolumn{2}{|l|}{ Hospitalization Findings } \\
\hline ICU patients & $8(17.0)$ \\
\hline Duration of symptoms before hospitalization (Mean, SD) & $10.7 \pm 5.0$ \\
\hline Hospitalization Duration (Mean, SD - Range, days) & $8.7 \pm 4.8(2-21)$ \\
\hline \multicolumn{2}{|l|}{ Chest CT-scan findings (Lung Involvement) } \\
\hline $10-25 \%$ & $9(19.1)$ \\
\hline $25-50 \%$ & $23(48.9)$ \\
\hline$>50 \%$ & $6(12.8)$ \\
\hline$>75 \%$ & $1(2.1)$ \\
\hline Missing data & $8(17.0)$ \\
\hline
\end{tabular}




\begin{tabular}{ll}
\hline Characteristics & Patients $(\mathrm{N}-\%)$ \\
\hline Biology Features & \\
Hemoglobin $(\mathrm{g} / \mathrm{dL})$ & $14.0 \pm 1.6$ \\
Neutrophils $\left(10^{\wedge} 3 / \mu \mathrm{l}\right)$ & $6.8 \pm 3.4$ \\
Lymphocytes $\left(10^{\wedge} 3 / \mu \mathrm{l}\right)$ & $1.1 \pm 0.5$ \\
Lymphopenia & $34(72.3)$ \\
Normopenia & $13(27.7)$ \\
Patelets $\left(10^{\wedge} 3 / \mu \mathrm{l}\right)$ & $242.9 \pm 113.2$ \\
CRP $(\mathrm{mg} / \mathrm{L})$ & $119.5 \pm 110.1$ \\
Creatinin $(\mathrm{mg} / \mathrm{dL})$ & $1.1 \pm 0.8$ \\
Bilirubin $(\mathrm{mg} / \mathrm{dL})$ & $0.5 \pm 0.3$ \\
D-Dimer $(\mu \mathrm{gg} / \mathrm{L})$ & $1258.0 \pm 531.1$ \\
LDH $(\mathrm{UI} / \mathrm{L})$ & $362.4 \pm 138.3$ \\
Na+ $(\mathrm{mmol} / \mathrm{L})$ & $136.9 \pm 3.6$ \\
K+ $(\mathrm{mmol} / \mathrm{L})$ & $4.1 \pm 0.7$ \\
Cl- $(\mathrm{mmol} / \mathrm{L})$ & $97.2 \pm 4.1$ \\
1-month Mean $(\mathrm{SD})$ IgG level & $173.3 \pm 80.6$ \\
\hline
\end{tabular}

Table 1 footnotes : Abbreviations: $\mathrm{CRP}=\mathrm{C}$-reactive Protein; $\mathrm{CT}=$ computed tomography; $\mathrm{GERD}=$ gastroesophageal reflux disease; $\mathrm{SD}=$ standard deviation.

Table 2: Olfactory Outcomes .

\begin{tabular}{ll}
\hline Olfactory Outcomes & \\
\hline Aroma Perception Disorder & $N=12$ \\
Total vs Partial loss of aroma perception sense & $1(2.1) / 6(12.8)$ \\
Distortion & $5(10.6)$ \\
Olfactory Outcomes & \\
Variable olfactory dysfunction & $5(44.4)$ \\
Nasal Obstruction related Dysfunction & $5(27.8)$ \\
Non-variable & $3(16.7)$ \\
Did not remember & $2(11.1)$ \\
Onset of Smell Dysfunction & $N=18$ \\
Before the other symptoms & $1(5.6)$ \\
Concomittant with other symptoms & $9(50.0)$ \\
After the other symptoms & $8(44.4)$ \\
Did not remember & $0(0)$ \\
Sniffin'sticks tests (Mean, SD) & $N=47$ \\
Mean value & $12.7 \pm 2.8$ \\
Anosmic & $4(8.5)$ \\
Hyposmic & $9(19.1)$ \\
Normosmic & $34(72.3)$ \\
SNOT-22 (Mean, SD) & $41.1 \pm 18.6$ \\
\hline
\end{tabular}

Table 2 footnotes : Abbreviations: $\mathrm{SD}=$ standard deviation; SNOT-22= sino-nasal outcome-22 questionnaire. 